\title{
Fast One-Step Ultrasensitive Detection of Toxocara canis Antigens by a Nanobody-Based Electrochemical Magnetosensor
}

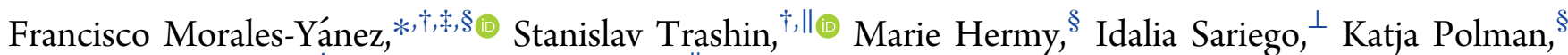
Serge Muyldermans, ${ }^{\ddagger}$ and Karolien De Wael ${ }^{\| \odot}$

${ }^{\ddagger}$ Laboratory of Cellular and Molecular Immunology, Vrije Universiteit Brussel, Brussels, Belgium

${ }^{\S}$ Department of Biomedical Sciences, Institute of Tropical Medicine, Antwerp, Belgium

"AXES Research Group, Department of Chemistry, University of Antwerp, Antwerp, Belgium

${ }^{\perp}$ Department of Parasitology, Institute of Tropical Medicine Pedro Kourí, Havana, Cuba

\section{Supporting Information}

\begin{abstract}
Human toxocariasis (HT) is a cosmopolitan zoonotic disease caused by the migration of the larval stage of the roundworm Toxocara canis. Current HT diagnostic methods do not discriminate between active and past infections. Here, we present a method to quantify Toxocara excretory/secretory antigen, aiming to identify active cases of $\mathrm{HT}$. High specificity is achieved by employing nanobodies (Nbs), single domain antigen binding fragments from camelid heavy chain-only antibodies. High sensitivity is obtained by the design of an electrochemical magnetosensor with an amperometric read-out. Reliable detection of TES antigen at 10 and $30 \mathrm{pg} / \mathrm{mL}$ level was demonstrated in phosphate buffered saline and serum, respectively. Moreover, the assay

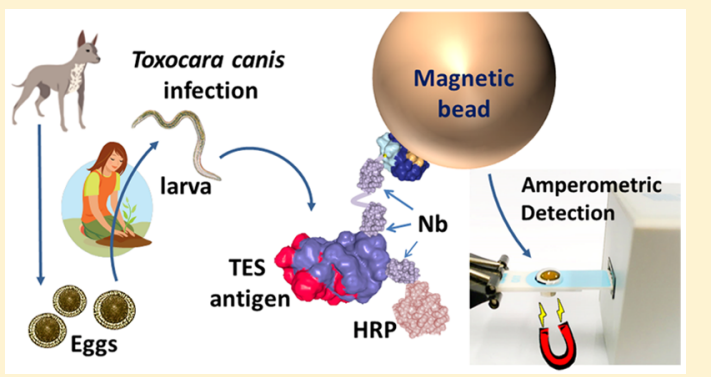
showed no cross-reactivity with other nematode antigens. To our knowledge, this is the most sensitive method to quantify the TES antigen so far. It also has great potential to develop point of care diagnostic systems in other conditions where high sensitivity and specificity are required.
\end{abstract}

$\mathrm{H}$ uman toxocariasis (HT) is the result of infection by the larval stages of Toxocara canis, a roundworm that colonizes the intestinal lumen of dogs. Upon infection, dogs excrete a huge amount of eggs in faeces that contaminate the soil of public spaces such as sandpits, playgrounds, and parks. Humans are infected by occasional ingestion of these eggs. In contrast to the final host, the parasite does not develop into the adult stage in humans. It remains as L2 larvae that may migrate toward the central nervous system, eyes, lungs and liver, provoking three clinical syndromes: the visceral larvae migrans, ocular larva migrans, and covert toxocariasis. ${ }^{1} \mathrm{HT}$ is a cosmopolitan disease found in developing ${ }^{2-4}$ and developed countries. $^{5-8}$ Despite its broad distribution over the world, the disease (especially in the form of covert toxocariasis) is frequently underdiagnosed.

Currently, diagnosis of human toxocariasis is based on serological analysis (i.e., the detection of specific immunoglobulins against the T. canis excretory/secretory antigens, TES) by enzyme-linked immunoassay (ELISA) and Western blot, combined with eosinophil counts in peripheral blood along with clinical and epidemiological evaluation. ${ }^{9}$ Cross-reactivity with other nematodes and the inability to distinguish between past and active infections are the main limitations of this approach. A more specific and sensitive diagnosis of active HT can be achieved through detection of TES antigen that is excreted by migrating larva. However, its low concentration in the bloodstream poses a major challenge. So far, only a few attempts to develop a TES antigen detection assay have been published. ${ }^{10-12}$ These assays rely on ELISA and polyclonal or monoclonal antibodies for TES antigen recognition, but suffer from the lack of sensitivity to detect trace concentrations of TES antigen in blood, which is the most important limitation of such assays.

Recently, nanobodies (Nbs) have emerged as cost-efficient and more stable immunoreagents to substitute monoclonal antibodies, but still remain largely unexplored in the field of analytical chemistry. Nbs are recombinant single-domain fragments of heavy chain antibodies from camelids (Figure 1A), which feature a convex binding site enabling recognition of concave-shaped epitopes that are normally cryptic to conventional antibodies with a large and shallow paratope. Besides their exceptional specificity, $\mathrm{Nbs}$ are easily identified and can be produced in Escherichia coli under standard protein production conditions, limiting the batch-to-batch variation observed for conventional monoclonal and polyclonal antibodies. ${ }^{13} \mathrm{Nbs}$ also serve as modular entities for a number of modifications (e.g., adding a tag or an enzyme label) that can be exploited for increasing sensitivity in assay development. ${ }^{14}$

Received: April 6, 2019

Accepted: August 20, 2019

Published: August 20, 2019 
A

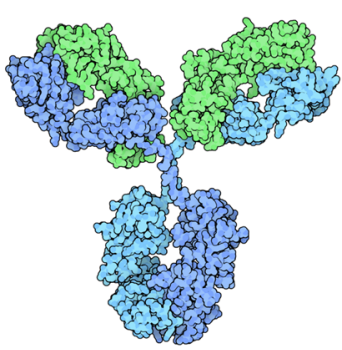

Conventional antibody (150 kDa)

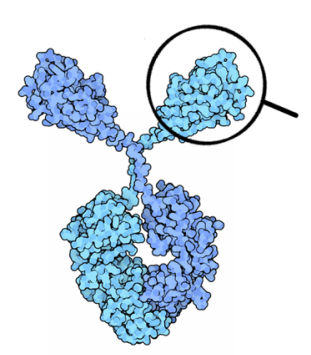

Heavy-chain

antibody from camelids

Single variable domain (Nanobody, Nb) 15 kDa

B

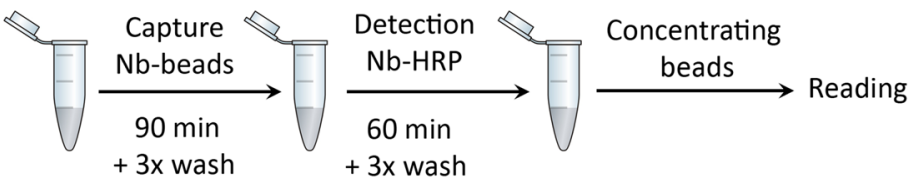

C Sample $+3 \mathrm{x}$ wash

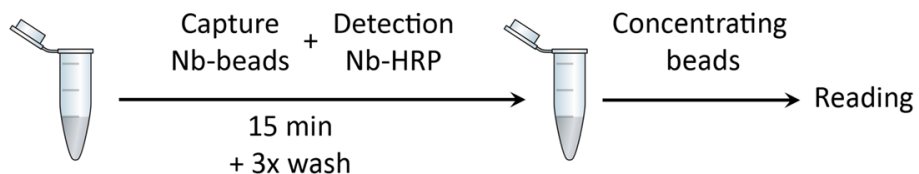

D

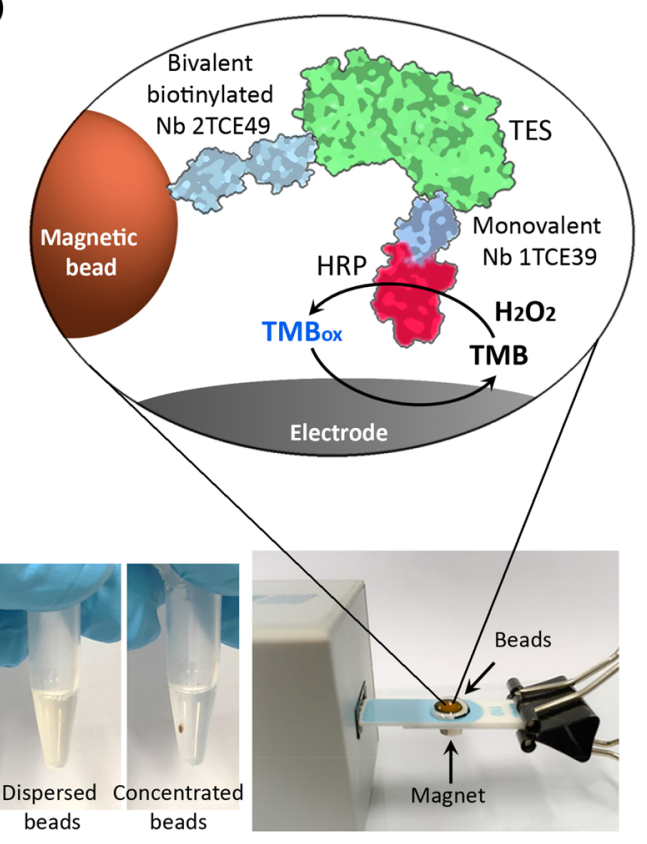

Figure 1. (A) Graphical representation of a conventional IgG antibody (left) and a heavy chain-only antibody (right) from camelids, where the antigen binding fragment is reduced to a single domain, the Nanobody. ${ }^{16} \mathrm{Nbs}$ are $10 \times$ smaller than $\operatorname{IgG}$ and can be produced in microbial expression systems. (B) A long procedure used for an electrochemical magnetic assay (magnetosensor), repeating the same scheme as the corresponding ELISA. (C) A short procedure with simultaneous adding capture and detection immunoreagents and a shortened incubation time. (D) During the electrochemical reading step, TMB undergoes the redox cycling, it is enzymatically oxidized in the presence of $\mathrm{H}_{2} \mathrm{O}_{2}$ and then electrochemically reduced at an electrode. (E) The magnetosensor setup is with concentrated beads placed on the electrode and plugged into a potentiostat for amperometric reading.

Our recent results ${ }^{15}$ indicated already a superior sensitivity and selectivity of a sandwich ELISA based on Nbs compared to conventional antibodies.

In this work, we address the need to further improve the sensitivity of the assay by using magnetic beads in combination with an amperometric readout in a configuration so-called electrochemical magnetosensor. ${ }^{17,18}$ The main difference between a magnetosensor and a biosensor is that the biorecognition probe is linked to magnetic beads that can be temporally dispersed in the analyte solution for improving binding kinetics and then accumulated on an electrode by a magnet to register an analytical response. In comparison with conventional optical read-out systems, electrochemical sensors can register an analytical response almost immediately and without the need for developing the reaction in bulk volume, since the response of electrochemical sensors is determined by a thin layer of measuring solution near the electrode surface where the beads with immunoreagents are placed. ${ }^{19}$ This approach may improve sensitivity and decreases analysis time in comparison to conventional ELISA. ${ }^{20}$

Previously, electrochemical biosensors have been extensively used in the diagnosis of tropical diseases. ${ }^{21}$ Relevant examples include malaria, ${ }^{22}$ Chagas' disease, ${ }^{23}$ tuberculosis, ${ }^{24}$ and schistosomiasis. $^{25}$ The electrochemical magnetosensor described here is, to our knowledge, the first attempt to detect TES antigen electrochemically, and we trust it is essential to achieve the sensitivity needed for the detection of low concentrations of TES antigen in body fluids.

\section{EXPERIMENTAL SECTION}

TES Antigen Production. Production and purification of TES antigen was carried out according to Savigny et al. ${ }^{26,27}$ The resulting material was reconstituted in sterile Milli-Q water, and the quality of the prepared TES antigen was confirmed by SDS PAGE (Supporting Information, Figure S1), which showed the typical band profile of the TES antigen. ${ }^{28}$ The concentration of TES antigen in the stock solution was determined in triplicate by NanoDrop (OD280 nm of 1 corresponds to $1 \mathrm{mg} / \mathrm{mL})$. In the literature, TES antigen concentration is often determined by a Bradford assay using BSA as a standard. TES antigen concentrations measured by NanoDrop score usually $7 \times$ higher than the concentration measured by Bradford assay. ${ }^{15}$ In addition, we evaluated cross reactivity of the assay with the following antigens: somatic (whole larvae extract) antigens obtained from third stage larva (L3) from Ascaris lumbricoides and Ascaris suum; excretory/ secretory and somatic antigens from Anisakis simplex and Pseudoterranova decipiens (kindly provided by Prof. Pierre Dorny from the Institute of Tropical Medicine Antwerp, Belgium). Additionally, we tested serum from a rat infected with Angiostrongylus cantonensis 42 days post-infection (where larvae are present and produce excretory/secretory antigens) and somatic antigens from the same parasite (kindly provided by Dr. Beatrice Nickel from the Swiss Institute of Tropical Medicine, Switzerland).

Library Construction and Selection of Nbs. Construction of $\mathrm{Nb}$ library, generation of $\mathrm{Nbs}$, and production of $\mathrm{Nbs}$ in E. coli cells was performed according to protocols developed in our laboratory. ${ }^{29-31} \mathrm{Nb}$ 2TCE49 and $\mathrm{Nb}$ 
1TCE39 were chosen to prepare capture and detection immunoreagents used in this work. The abbreviations of these $\mathrm{Nbs}$ correspond to those used in our previous work, where the specificity of a series of TES antigen specific Nbs are discussed in detail. ${ }^{15}$

Nb Production. Nb 2TCE49 was expressed in pBAD-17 vector (containing an AVI-tag) ${ }^{32}$ and coexpressed with pACYC184 plasmid (Avidity, U.S.A.) containing $\mathrm{pBirA}_{C m}$ enzyme for in vivo biotinylation and used as biotinylated bivalent-monospecific construct. Purification of biotinylated Nbs was performed by affinity chromatography on a Streptavidin-Mutein Matrix (Roche) and subsequent sizeexclusion chromatography (SEC) on Äkta Explorer (GE Healthcare Life Sciences) using a Superdex 75 HR 10/30 column (GE Healthcare Life Sciences) in PBS. Biotinylation was confirmed by SDS-PAGE of $\mathrm{Nbs}$ under reducing conditions followed by transfer onto a nitrocellulose membrane and coloring with horseradish peroxidase (HRP) coupled to streptavidin (Biolegend, U.S.A.) diluted 1:2000. Monovalent biotinylated construct 2TCE49 was used as control to compare molecular weight. A picture of the corresponding blot membrane is given in the (Supporting Information, Figure S2).

The second selected $\mathrm{Nb}$ ( $\mathrm{Nb}$ 1TCE39), which shows a different binding profile to TES antigen compared to the antigen capturing $\mathrm{Nb}$ 2TCE49, was expressed from pHEN6c vector containing only His-Tag. After purification by SEC, it was chemically coupled with HRP using a commercial kit (Abcam, United Kingdom). Due to the small size of Nbs in comparison to standard monoclonal antibodies, we adapted the conjugation protocol to a molecular weight of $15 \mathrm{kDa}$.

Sandwich ELISA. ELISA plates were coated with streptavidin (Sigma-Aldrich) overnight at room temperature. After washing, $100 \mu \mathrm{L}$ of biotinylated-bivalent capture $\mathrm{Nb}$ diluted in PBS-0.05\% Tween-20 (PBS-T20) was added and incubated for $1 \mathrm{~h}$ at room temperature. Next, the plates were blocked with $200 \mu \mathrm{L}$ of PierceProtein-free blocking buffer (Thermofisher Scientific) for $2 \mathrm{~h}$. Then, the plates were washed and TES antigen standard solutions spiked in PBS-T20 or serum were added to the wells. After $90 \mathrm{~min}$ of incubation at room temperature and washing, detection of $\mathrm{Nb}-\mathrm{HRP}$ conjugate was added and incubated for $1 \mathrm{~h}$. Finally, a twopart $3,3^{\prime}, 5,5^{\prime}$-tetramethylbenzidine $(\mathrm{TMB})$ substrate kit reagent OptEIA (BD) was added to the wells, and the reaction developed for $20 \mathrm{~min}$ before reading results.

The limit of detection (LOD) was calculated as the concentration giving a response that equals the average plus three times the standard deviations of six blanks (PBS-T20 or serum).

Electrochemical Magnetosensor. The electrochemical magnetosensor was set up with the same immunoreagents as used in ELISA. A neodymium magnet was used to accumulate the magnetic beads in microtubes during washing steps and at the electrode for recording responses.

First, streptavidin-modified magnetic beads (Dynabeads M280, ThermoFisher Scientific) were washed and coated with biotinylated-bivalent Nb2TCE49 $(2-5 \mu \mathrm{g} / \mathrm{mL}$ in PBS-T20 to get a coverage of $2 \mu \mathrm{g} \mathrm{Nb}$ per mg beads) on a rotary shaker for $1 \mathrm{~h}$ at room temperature. Next, the beads were washed with PBS-T20 and a fraction of $50 \mu \mathrm{g}$ was taken for each analysis. A long and a short procedure was tested for TES antigen detection (Figure 1B,C). In the long procedure, the beads were incubated with the antigen for $90 \mathrm{~min}$ (volume $500 \mu \mathrm{L}$ ), followed by triple washings with PBS-T20 and $60 \mathrm{~min}$ incubation with detection Nb-HRP conjugate. This procedure contains the same time intervals as used in the ELISA protocol. In the short procedure, the coated beads and the Nb-HRP were added simultaneously to the antigen solution and incubated for only $15 \mathrm{~min}$. Prior to the electrochemical reading, the beads were washed $3 \times$ with PBS-T20 and stored in PBS.

Just before electrochemical reading, the beads were resuspended in $15 \mu \mathrm{L}$ of $0.5 \mathrm{mM} \mathrm{TMB}$ in citrate-acetate buffer ( $35 \mathrm{mM}$ sodium acetate, $50 \mathrm{mM}$ citric acid, $\mathrm{pH}$ 5.0) and injected in a drop of $60 \mu \mathrm{L}$ reading buffer ( $0.5 \mathrm{mM} \mathrm{TMB}, 2$ $\mathrm{mM} \mathrm{H} \mathrm{H}_{2} \mathrm{O}_{2}$ in the citrate-acetate buffer $\mathrm{pH} 5.0$ ) on a carbon screen-printed electrode (SPE, Dropsens). A neodymium magnet was placed below SPE to accumulate the beads at the surface of the electrode (Figure 1B). The current was registered for $180 \mathrm{~s}$ by a potentiostat EmStat Blue (PalmSens) and the average current of the last $10 \mathrm{~s}$ was taken as the result of the electrochemical reading. PBS-T20 and serum without TES antigen were used as blanks. The LOD was calculated from an average signal of four blanks plus three times the standard deviation. A graphical representation of the assay is depicted in Figure 1.

Data Analysis. Standard curves were constructed and estimated through 5PL model.

\section{RESULTS AND DISCUSSION}

Comparison between ELISA and the Electrochemical Magnetosensor Assay. In contrast to the conventional ELISA, the biorecognition element of an electrochemical magnetosensor consists of modified magnetic beads that specifically recognize a target analyte. For the analysis, the beads are dispersed into a sample and, afterward, washed and concentrated by a magnet to be placed at the sensor surface. This detection scheme can be more sensitive and rapid compared to conventional ELISA and biosensors with a capture immunoreagent confined at a sensor surface. ${ }^{33}$

Previously, we developed an ELISA for the quantification of TES antigen based on biotinylated bivalent Nbs and HRPconjugated detection Nbs. ${ }^{15}$ Here, we could directly compare the ELISA and the electrochemical magnetosensor assay since biotinylated $\mathrm{Nbs}$ can be attached to commercially available streptavidin-coated beads, while the presence of detection $\mathrm{Nb}$ (Nb-HRP conjugate) can be measured amperometrically due to electrochemical reduction of an oxidized form of TMB in the conventional measuring solution, ${ }^{34}$ as in Figure 1D. In both electrochemical and optical assays, a biotinylated bivalent construct was used as the capture $\mathrm{Nb}$ to reduce a possible steric factor of the support.

For clear comparison between ELISA and the magnetosensor assay we applied the same sequence of the steps and the same time intervals (Figure 1B), that is, 90 min reaction with the antigen, followed by an additional $60 \mathrm{~min}$ incubation with detection Nbs. However, the measuring step was different. In ELISA, reading required developing of the HRP-catalyzed reaction (oxidation of TMB by $\mathrm{H}_{2} \mathrm{O}_{2}$ with the accumulation of the colored product) over $20 \mathrm{~min}$ and quenching the reaction with sulfuric acid $0.2 \mathrm{M}$, while the amperometric reading with the magnetosensor was executed immediately after the beads were transferred onto the electrode. The instant reading at an electrode is possible due to the close proximity of the sensing surface to the detection $\mathrm{Nb}$ that results in the redox cycling of 
the oxidized and reduced forms of TMB between the electrode and HRP (Figure 1D).

When using the two-part substrate reagent set, we have experienced irregular noise in blanks during amperometric reading (data not shown), probably due to a high concentration of methanol in the reagent $\mathrm{B}(1 \mathrm{mM}$ TMB in $33 \%$ methanol-water mixture), which can affect the conductive inks in SPE electrodes. Thus, we replaced the kit reagent by $25 \mathrm{mM}$ TMB solution in DMSO, keeping the same $\mathrm{pH}$ (citrate-acetate buffer $\mathrm{pH}$ 5.0) and the final concentrations of the components $\left(2 \mathrm{mM} \mathrm{H}_{2} \mathrm{O}_{2}\right.$ and $\left.0.5 \mathrm{mM} \mathrm{TMB}\right)$. This completely solved the problem.

Calibration curves for both the ELISA and the electrochemical magnetic immunoassay are given in Figure 2. The
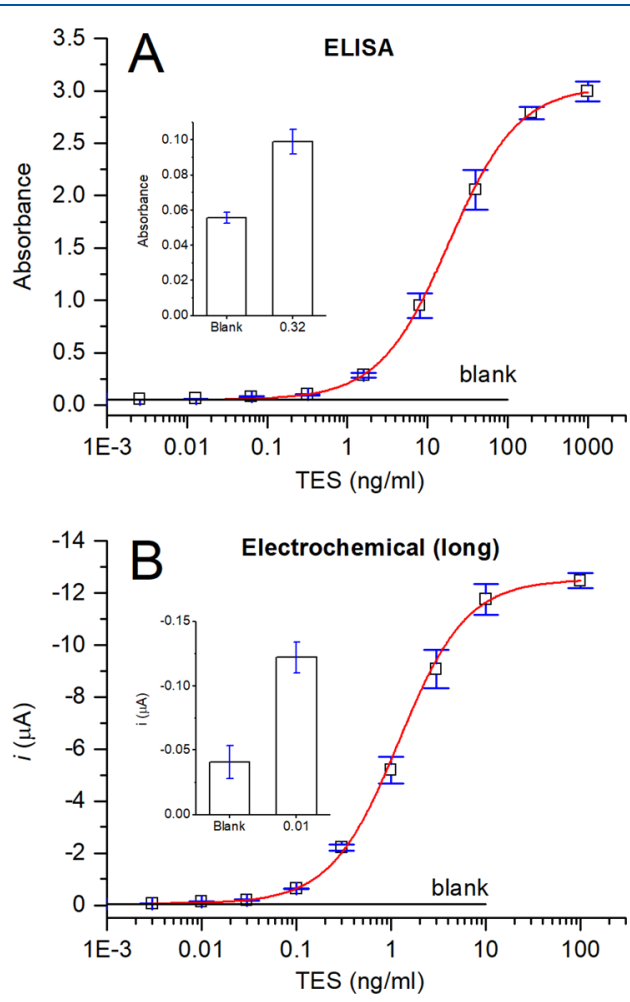

Figure 2. Calibration curve for detecting TES antigen in PBS-T20 via a sandwich ELISA (A) and magnetosensor assay (B) using the same immunoreagents and incubation time ("long" procedure, Figure 1B). Black lines indicate the average response of blanks. Potential applied, $-0.1 \mathrm{~V}$.

LOD for ELISA was $90 \mathrm{pg} / \mathrm{mL}$ in PBS-T20 (interpolated to the response of blanks plus $3 \mathrm{xSD}$ ) or $320 \mathrm{pg} / \mathrm{mL}$ as the first experimentally detected concentration above the level of blanks plus 3xSD (Figure 2A). Under the same conditions, the electrochemical magnetosensor assay had an LOD of $6 \mathrm{pg} /$ $\mathrm{mL}$ ( or $10 \mathrm{pg} / \mathrm{mL}$ experimentally detected above the level of blanks plus $3 \mathrm{xSD}$, Figure $2 \mathrm{~B}$ ). The limitation of the maximal response by approximately $10-12 \mu \mathrm{A}$ at concentrations above $10 \mathrm{ng} / \mathrm{mL}$ likely resulted from rapid complete oxidation of $\mathrm{TMB}$ at the beads and the overall limitation of the reaction by diffusion of TMB between the electrode and the peroxidise enzyme on the beads.

Optimization of Electrochemical Assay. We selected two Nbs belonging to two different families, each of which recognize an independent epitope on the TES antigen, as capturing and detection Nbs. Thus, we could significantly decrease the analysis time by incubating the analyte with the capture and detection Nbs simultaneously (Figure 1C). Moreover, the duration of the biorecognition step should be noticeably decreased due to enhanced diffusion of the antigen with a high molecular weight toward the beads dispersed in the sample compared to diffusion of such antigen to the surface of wells in ELISA microtiter plates.

To minimize the analysis time, we applied the short detection scheme (Figure 1C) and varied incubation time between 10 and $90 \mathrm{~min}$ (Supporting Information, Figure S3). The results suggest that a short incubation of $15 \mathrm{~min}$ was sufficient to complete the binding step of TES antigen at $1 \mathrm{ng} /$ $\mathrm{mL}$, because responses did not increase for prolonged incubation times. A shorter incubation time of $10 \mathrm{~min}$, however, was not sufficient and showed a reduced average response (by 20\%) and an increased standard deviation. This was likely due to difficulties to exactly reproduce this rather short incubation time taking into account handling the samples during adding the reagents and washing steps. Thus, an incubation of $15 \mathrm{~min}$ was preferred in the following experiments.

The shortening of the analysis procedure opens an opportunity for creation of a point of care (POC) diagnostic test requiring minimum equipment and a total analysis time as low as $30 \mathrm{~min}$ per individual sample.

Next, we investigated the effect of the applied potential on the recorded signal. Optimal potential was found between -0.1 and $0.0 \mathrm{~V}$ giving maximum sensitivity at acceptably low blanks (Figure 3). The potential of $-0.1 \mathrm{~V}$ was used in the following experiments.

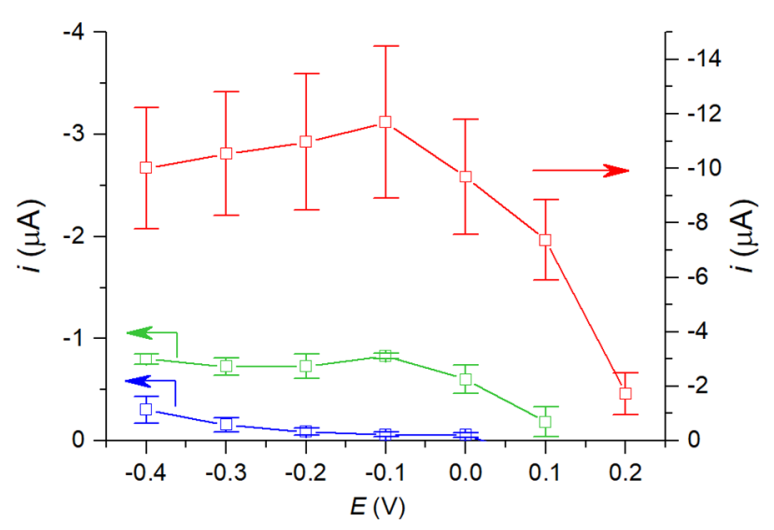

Figure 3. Dependence of the current on the potential applied in measurements with the magnetosensor at $100 \mathrm{ng} / \mathrm{mL}$ of TES antigen (red, the current is shown on the right-side axis), $0.1 \mathrm{ng} / \mathrm{mL}$ of TES antigen (green), and blank PBS-T20 (blue). Incubation time, $15 \mathrm{~min}$; beads per measurement, $50 \mu \mathrm{g}$.

Then, we evaluated the effect of the amount of beads used per analysis that is eventually transferred onto the electrode for a single reading (Figure 4). We observed that $50 \mu \mathrm{g}$ of beads is enough for detection of TES antigen, but if needed, this amount can be decreased about twice to reduce the consumption of the reagent without compromising the signal intensity. We used $50 \mu \mathrm{g}$ of beads to ensure a good sensitivity and reproducibility even if a part of the reagent is lost during the analysis

Finally, the effect of the concentration of detection Nbs was estimated at $5 \mathrm{ng} / \mathrm{mL}$ concentration of TES antigen. The response was virtually independent of the amount of the 


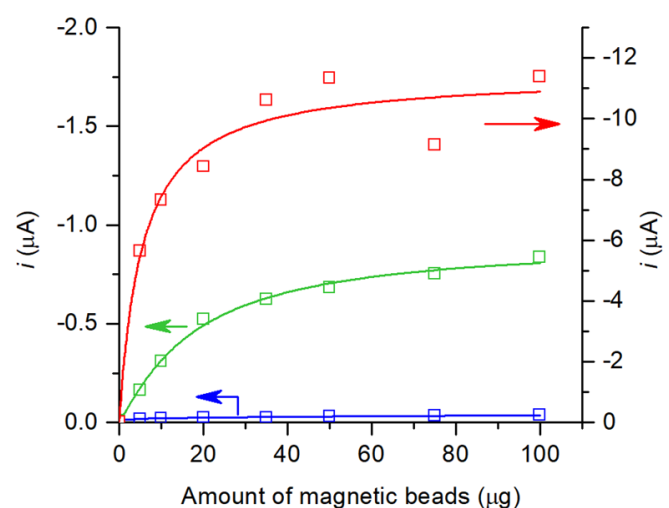

Figure 4. Effect of the magnetic beads quantity used per analysis on the amperometric response to $100 \mathrm{ng} / \mathrm{mL}$ (red, the current is shown on the right-side axis), $0.1 \mathrm{ng} / \mathrm{mL}$ TES antigen (green) diluted in PBS-T20 in comparison to blank PBS-T20 (blue). Potential applied, $-0.1 \mathrm{~V}$.

detection $\mathrm{Nbs}$ in the range of $5-100 \mathrm{ng} / \mathrm{mL}$ (Supporting Information, Figure S4) and was kept at $10 \mathrm{ng} / \mathrm{mL}$ in the following experiments to reduce the consumption of the reagent.

Detection of TES Antigen in Serum. After optimization of critical parameters, we tested the electrochemical magnetic assay in PBS-T20 and serum spiked with TES antigen. The one-step assay in PBS-T20 performed as good as the assay with two steps (Figure 1C) and could reliably detect $10 \mathrm{pg} / \mathrm{mL}$ of TES in PBS-T20 (Figure 6A). However, the detectability of TES antigen spiked in serum was impeded, which was likely due to the matrix effect provoked by the complex composition of serum. At $1 \mathrm{ng} / \mathrm{mL}$ of TES antigen, the response in serum was only $62 \%$ of the response in PBS-T20 (Figure 5). The

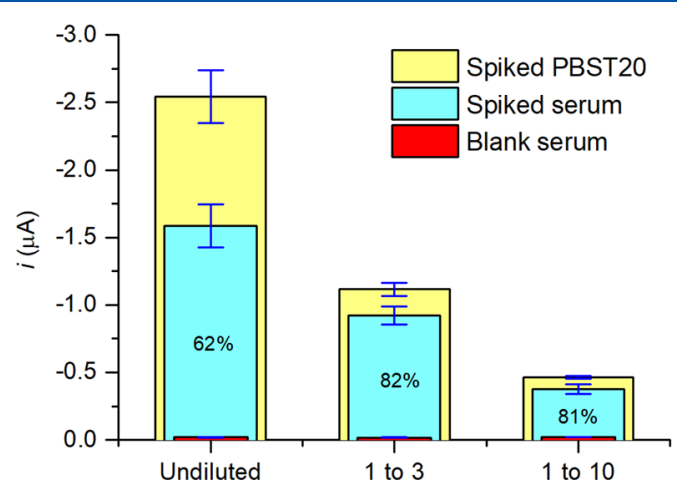

Figure 5. Dilution effect for PBS-T20 and serum containing $1 \mathrm{ng} / \mathrm{mL}$ of TES antigen using PBS-T20 as diluent. Percentage values depict the responses in serum relative to PBS-T20.

matrix effect was minimized upon diluting the serum three to ten times, which then resulted in only $20 \%$ loss of the response. However, the dilution proportionally decreases the effective concentration of the antigen in the measured solution. Thus, measurement of TES antigen in serum appeared to be more efficient and could provide the highest sensitivity.

Due to the matrix effect, the calibration curve for the electrochemical magnetosensor in serum showed lower responses through the whole concentration range compared to PBS-T20 resulting in a 3 -fold higher LOD with a reliable detection of TES antigen at $30 \mathrm{pg} / \mathrm{mL}$ in serum as demonstrated experimentally (Figure $6 \mathrm{~A}$ ). Compared to the
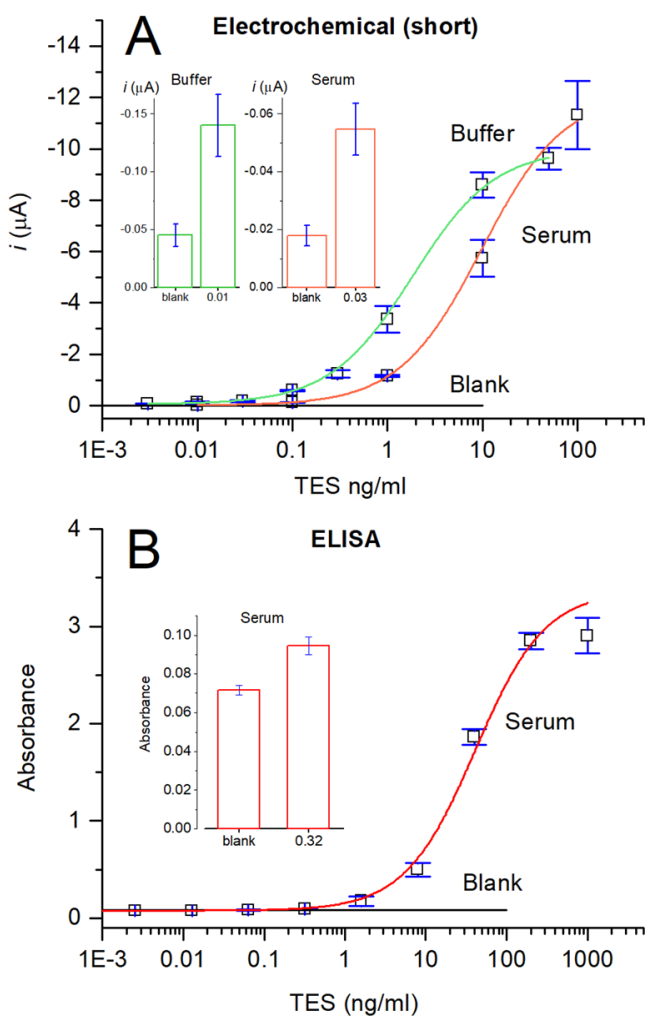

Figure 6. (A) Detection of TES antigen in PBS-T20 and serum using an amperometric magnetosensor and a shortened protocol, as in Figure 1C. (B) Calibration curve for ELISA in serum spiked with TES antigen. Black line indicates the average of blanks.

optimized electrochemical assay, the nanobody-based ELISA in serum performed considerably worse with the LOD of only $200 \mathrm{pg} / \mathrm{mL}$ in serum (interpolated to the average of blanks plus $3 \mathrm{xSD}$ ) or experimentally demonstrated $320 \mathrm{pg} / \mathrm{mL}$ that was reliably detected in serum (Figure 6B). Other ELISAs using classical polyclonal and/or monoclonal antibodies for TES antigen detection reported even worse LOD values of $20,{ }^{10} 5,{ }^{11}$ and $0.440 \mathrm{ng} / \mathrm{mL}$ (in buffer). ${ }^{12}$

The electrochemical magnetosensor assay performed 2-3 orders of magnitude better than the ELISAs reported in the literature and 10-20× better than our nanobody-based ELISA, with the superior immunoreagents elaborated previously in our group. ${ }^{15}$ Moreover, the analysis time with the electrochemical magnetosensor assay was reduced to only $30 \mathrm{~min}$ when measuring a few individual samples and without counting the coating step (additional $60 \mathrm{~min}$ ). The coating step can be avoided in practice by preparing stable covalently modified beads with capture Nbs and applying them as a ready-to-use reagent. $^{35}$ This is technically possible due to the high stability of Nbs and their excellent shelf life (stable for months at $4{ }^{\circ} \mathrm{C}$ without losing antigen binding efficiency). ${ }^{36}$ Thus, the short time of analysis, the high sensitivity, and the use of a cheap and simple amperometric readout fulfilled the important requirements for the development of POC diagnosis of HT. Moreover, the $\mathrm{Nb}$ electrochemical assay described here demonstrated the highest analytical sensitivity for TES antigen detection currently achieved.

Achieving a detection level within the $\mathrm{pg} / \mathrm{mL}$ range is a critical factor to diagnose the active stage of the disease. A recent study ${ }^{37}$ using a mouse model of inoculation of T. canis eggs showed that the concentration of TES antigen in serum 
from mice infected with 50 eggs ranged from 0.013 to $0.49 \mu \mathrm{g} /$ $\mathrm{mL}$ (quantified by Bradford assay) or $0.09-3.43 \mu \mathrm{g} / \mathrm{mL}$ (recalculated to the concentrations quantified by absorbance at $280 \mathrm{~nm}$ with NanoDrop spectrophotometer as used in our work). If we extrapolate these numbers in mice, having $1.5 \mathrm{~mL}$ of blood on average, to an infection of 50 eggs in an adult human having on average of $5 \mathrm{~L}$ of blood, we obtain a concentration of TES antigen in the range from 0.02 to 0.69 $\mathrm{ng} / \mathrm{mL}$ of serum, which lies well within the range of our electrochemical assay.

Although the mice model does not reproduce the real conditions of $T$. canis infections in humans due to the large amount of inoculum compared to the size of the experimental animals, the estimated values roughly agree with the estimations made from the amount of TES antigen produced by $T$. canis larva per day. Badley et al. estimated that a larva produces about 8 ng of protein per day. ${ }^{38}$ This amount of TES antigen is released on a daily basis into the tissues of a patient. Approximately $60 \%$ of an adult human body mass is an aqueous solution of ions and other substances, and one-third of it is outside the cells in what is called the extracellular fluid. ${ }^{39}$ In an adult of $75 \mathrm{~kg}$, that represents approximately $15 \mathrm{~L}$ of liquid in which TES antigen is dissolved. Assuming an inoculum of 50-200 larvae producing in total 400 to $1600 \mathrm{ng}$ of TES antigen per day that reaches the extracellular compartment, the concentrations of TES antigen in serum should range between 26 and $106 \mathrm{pg} / \mathrm{mL}$, without taking into account the material that remains inside the granulomas, extracellular tissues, or neutralized by the immune system of the host. Notably, the amount of TES antigen produced by larvae in vitro depends on maintaining conditions (medium used, number of larvae per $\mathrm{mL}$ of medium taken, etc.), leading to a high variation in the estimations (e.g., $10 \mathrm{pg} / \mathrm{larva} /$ day $^{40}$ and $2 \mathrm{ng} / \mathrm{larva} / \mathrm{day}^{41}$ ), while the exact amount of TES antigen excreted in real infection conditions is essentially unclear. Nevertheless, we expect that the clinically relevant concentration of TES antigen in serum will be in the range of $0.01-1$ $\mathrm{ng} / \mathrm{mL}$ serum, which is in consensus with either way of estimation when considering an infection by 50-200 eggs of the parasite.

Our electrochemical magnetic assay has an LOD of $10 \mathrm{pg} /$ $\mathrm{mL}$ in serum, which means it has the potential to spot relevant HT active infections in the form of covert toxocariasis. This degree of sensitivity has never been achieved by any other TES antigen detection test and makes our assay a very promising strategy to detect the antigen in a clinical or epidemiological context. Conversely, ultrasensitive detection of TES antigen would not only help to distinguish between active and past Toxocara infections, but it also might be a key component in the understanding of the dynamics of TES antigen concentration in serum and antibody response in vivo.

Cross-Reactivity with Other Parasites. HT is a parasitic condition that is more prevalent in tropical countries, where polyparasitism is frequent. This represents a challenge for the diagnosis of the disease because T. canis shares some epitopes with other parasites such as Ascaris lumbricoides and Ascaris suum. ${ }^{42,43}$ To determine the cross-reactivity of the test to other helminths, we evaluated the optimized electrochemical assay with excretory/secretory (ES) and somatic antigens from five other nematodes. The response to this battery of antigens at a concentration of $1 \mathrm{ng} / \mathrm{mL}$ did not differ from blank, confirming the high specificity of the assay (Figure 7).

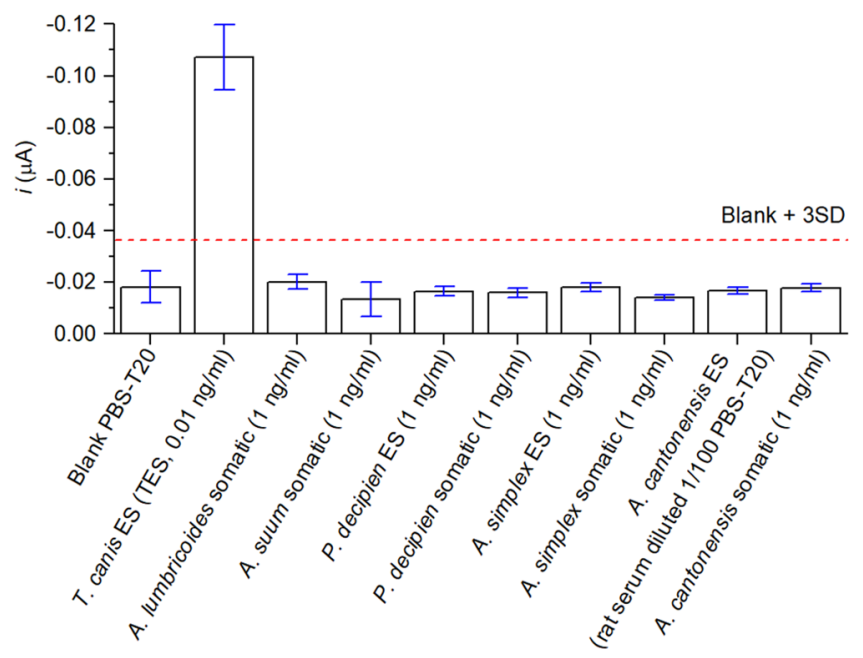

Figure 7. Specificity of the electrochemical magnetosensor toward Toxocara canis (TES) antigen in comparison to excretory/secretory (ES) and somatic antigens of five other parasites: Angiostrongylus cantonenis, Ascaris lumbricoides, Ascaris suum, Pseudoterranova decipiens, and Anisakis simplex. The full set of antigens were tested at a concentration of $1 \mathrm{ng} / \mathrm{mL}$. Blank PBS-T20 and $0.01 \mathrm{ng} / \mathrm{mL}$ of TES antigen were used as negative and positive controls. Incubation time, $15 \mathrm{~min}$; potential applied, $-0.1 \mathrm{~V}$.

Noteworthy, surprisingly little attention has been paid so far to nanobodies in the field of biosensors, despite their high affinity, excellent stability, ease of purification, low cost (due to production in bacteria), and the possibility of using the molecular biology toolbox for their engineering and modification. In this work, we demonstrated the great potential of nanobodies as a biorecognition element in electrochemical (bio)sensors and addressed the need for lowering the limit of detection in conventional ELISA.

The electrochemical magnetosensor presented here showed the highest sensitivity and specificity compared to other diagnostic systems currently available for TES antigen. Moreover, the demonstrated detection strategy based on the magnetosensor in combination with a bivalent (or biparatopic) $\mathrm{Nb}$ can be easily adapted for analysis of many other targets where endogenous antibodies may complicate the target detection, and high sensitivity is crucial (e.g., viruses, presence of venoms in intoxicated patients, etc.).

\section{ASSOCIATED CONTENT}

\section{S Supporting Information}

The Supporting Information is available free of charge on the ACS Publications website at DOI: 10.1021/acs.analchem.9b01687.

SDS PAGE of TES antigen and biotinylated monovalent and bivalent Nbs. Effect of the incubation time and concentration of detection $\mathrm{Nb}$ on the magnetosensor response (PDF).

\section{AUTHOR INFORMATION}

\section{Corresponding Author}

*E-mail: francisco.morales.yanez@vub.be; franmorales11@ yahoo.com.ar.

ORCID $\odot$

Francisco Morales-Yánez: 0000-0002-2297-1479

Stanislav Trashin: 0000-0002-4089-4251 
Karolien De Wael: 0000-0003-4495-0748

\section{Author Contributions}

${ }^{\dagger}$ These authors contributed equally. The manuscript was written through contributions of all authors. All authors have given approval to the final version of the manuscript.

\section{Notes}

The authors declare no competing financial interest.

\section{ACKNOWLEDGMENTS}

This project was funded by the Fonds Wetenschappelijk Onderzoek-Vlaanderen (FWO-Flanders, Project G.0189.13N) and BOF UAntwerp. The authors acknowledge Prof. Pierre Dorny (Institute of Tropical Medicine Antwerp) and Dr. Beatrice Nickel (Swiss Institute of Tropical Medicine) for providing the antigens needed for the cross-reactivity experiments.

\section{REFERENCES}

(1) Glickman, L. T.; Schantz, P. M. Epidemiol. Rev. 1981, 3, 230250.

(2) Magnaval, J. F.; Michault, A.; Calon, N.; Charlet, J. P. Trans. $R$. Soc. Trop. Med. Hyg. 1994, 88, 531-533.

(3) Schoenardie, E. R.; Scaini, C. J.; Brod, C. S.; Pepe, M. S.; Villela, M. M.; McBride, A. J.; Borsuk, S.; Berne, M. E. J. Parasitol. 2013, 99, 537-539.

(4) Alvarado-Esquivel, C.; Hernandez-Tinoco, J.; Sanchez-Anguiano, L. F. J. Clin. Med. Res. 2015, 7, 176-181.

(5) Stensvold, C. R.; Skov, J.; Moller, L. N.; Jensen, P. M.; Kapel, C. M.; Petersen, E.; Nielsen, H. V. Clin. Vaccine Immunol. 2009, 16, 1372-1373.

(6) Buijs, J.; Borsboom, G.; van Gemund, J. J.; Hazebroek, A.; van Dongen, P. A.; van Knapen, F.; Neijens, H. J. Am. J. Epidemiol. 1994, 140, 839-847.

(7) Nijsse, R.; Ploeger, H. W.; Wagenaar, J. A.; Mughini-Gras, L. Parasitol. Res. 2015, 114, 561-569.

(8) Mughini-Gras, L.; Harms, M.; van Pelt, W.; Pinelli, E.; Kortbeek, T. Parasitol. Res. 2016, 115, 3779-3794.

(9) Fillaux, J.; Magnaval, J. F. Vet. Parasitol. 2013, 193, 327-336.

(10) Robertson, B. D.; Burkot, T. R.; Gillespie, S. H.; Kennedy, M. W.; Wambai, Z.; Maizels, R. M. Clin. Exp. Immunol. 1988, 74, 236241.

(11) Ishiyamna, S.; Ono, K.; Rai, S. K.; Uga, S. Nepal Med. Coll. J. 2009, 11, 9-13.

(12) Rodriguez-Caballero, A.; Martinez-Gordillo, M. N.; MedinaFlores, Y.; Medina-Escutia, M. E.; Meza-Lucas, A.; Correa, D.; Caballero-Salazar, S.; Ponce-Macotela, M. Parasites Vectors 2015, 8, 264-230.

(13) Voskuil, J. F1000Research 2014, 3, 232.

(14) Schumacher, D.; Helma, J.; Schneider, A. F. L.; Leonhardt, H.; Hackenberger, C. P. R. Angew. Chem., Int. Ed. 2018, 57, 2314-2333.

(15) Morales-Yanez, F. J.; Sariego, I.; Vincke, C.; HassanzadehGhassabeh, G.; Polman, K.; Muyldermans, S. Int. J. Parasitol. 2019, 49, 635-645.

(16) Goodsell, D. S. RCSB Protein Data Bank 2011, na

DOI: $10.2210 / \mathrm{rcsb} \_p d b / \mathrm{mom} \_2011 \_4$.

(17) Zacco, E.; Pividori, M. I.; Alegret, S.; Galve, R.; Marco, M. P. Anal. Chem. 2006, 78, 1780-8.

(18) Zacco, E.; Adrian, J.; Galve, R.; Marco, M. P.; Alegret, S.; Pividori, M. I. Biosens. Bioelectron. 2007, 22, 2184-2191.

(19) Richardson, J.; Hawkins, P.; Luxton, R. Biosens. Bioelectron. 2001, 16, 989-993.

(20) Croci, L.; Delibato, E.; Volpe, G.; Palleschi, G. Anal. Lett. 2001, 34, 2597-2607.

(21) Campuzano, S.; Yáñez-Sedeño, P.; Pingarrón, J. M. ChemElectroChem 2017, 4, 753-777.
(22) de Souza Castilho, M.; Laube, T.; Yamanaka, H.; Alegret, S.; Pividori, M. I. Anal. Chem. 2011, 83, 5570-7.

(23) Ferreira, A. A.; Colli, W.; da Costa, P. I.; Yamanaka, H. Biosens. Bioelectron. 2005, 21, 175-181.

(24) Diaz-Gonzalez, M.; Gonzalez-Garcia, M. B.; Costa-Garcia, A. Biosens. Bioelectron. 2005, 20, 2035-2043.

(25) Mach, K. E.; Mohan, R.; Patel, S.; Wong, P. K.; Hsieh, M.; Liao, J. C. PLoS Neglected Trop. Dis. 2015, 9, e0003845.

(26) de Savigny, D. H. J. Parasitol. 1975, 61, 781.

(27) Thomas, D.; Jeyathilakan, N.; Abdul Basith, S.; Senthilkumar, T. M. J. Parasit. Dis. 2016, 40, 1038-1043.

(28) Kennedy, W. M. Toxocara: The Enigmatic Parasite; CABI Publishing: Oxfordshire, 2006.

(29) Conrath, K. E.; Lauwereys, M.; Galleni, M.; Matagne, A.; Frere, J. M.; Kinne, J.; Wyns, L.; Muyldermans, S. Antimicrob. Agents Chemother. 2001, 45, 2807-2812.

(30) Vincke, C.; Gutiérrez, C.; Wernery, U.; Devoogdt, N.; Hassanzadeh-Ghassabeh, G.; Muyldermans, S. Generation of single domain antibody fragments derived from camelids and generation of manifold constructs. In Antibody Engineering. Methods in Molecular Biology (Methods and Protocols); Humana Press: Totowa, 2012; Vol. 907, pp 145-176.

(31) Pardon, E.; Laeremans, T.; Triest, S.; Rasmussen, S. G.; Wohlkonig, A.; Ruf, A.; Muyldermans, S.; Hol, W. G.; Kobilka, B. K.; Steyaert, J. Nat. Protoc. 2014, 9, 674-693.

(32) Beckett, D.; Kovaleva, E.; Schatz, P. J. Protein Sci. 1999, 8, 921-929.

(33) Rusling, J. F.; Kumar, C. V.; Gutkind, J. S.; Patel, V. Analyst 2010, 135, 2496-2511.

(34) Bos, E. S.; van der Doelen, A. A.; Rooy, N. v.; Schuurs, A. H. W. M. J. Immunoassay 1981, 2, 187-204.

(35) Even-Desrumeaux, K.; Fourquet, P.; Secq, V.; Baty, D.; Chames, P. Mol. BioSyst. 2012, 8, 2385-2394.

(36) Muyldermans, S. Annu. Rev. Biochem. 2013, 82, 775-97.

(37) Rodriguez-Caballero, A.; Martinez-Gordillo, M. N.; CaballeroSalazar, S.; Rufino-Gonzalez, Y.; Ponce-Macotela, M. Vet. Parasitol. 2017, 243, 183-187.

(38) Badley, J. E.; Grieve, R. B.; Bowman, D. D.; Glickman, L. T.; Rockey, J. H. J. Parasitol. 1987, 73, 593.

(39) Hall, J. E. Guyton and Hall Textbook of Medical Physiology, 13th ed.; Saunders: Philadelphia, 2016.

(40) de Savigny, D. H.; Tizard, I. R. Trans. R. Soc. Trop. Med. Hyg. 1977, 71, 501-507.

(41) Bowman, D. D.; Mika-Grieve, M.; Grieve, R. B. Am. J. Trop. Med. Hyg. 1987, 36, 75-82.

(42) Niel, G.; Pinon, J. M.; Couture, J.; Rosin, G.; Gentilini, M. Bull. Soc. Pathol. Exot. Ses Fil. 1973, 66, 324-332.

(43) Magnaval, J. F.; Glickman, L. T.; Grieve, R. B.; Carriere, E. D.; Dubly, E. Bull. Soc. Pathol. Exot. Ses Fil. 1986, 79, 634-641. 